\section{Endoscopic sinus surgery for paranasal sinus mucocoele with orbital involvement}

JJ Khong ${ }^{1}$, R Malhotra' ${ }^{1}$, PJ Wormald ${ }^{2}$ and D Selva ${ }^{1}$
Keywords: frontal mucocoele; frontoethmoidal mucocoele; endoscopic sinus surgery; ESS; orbit

\section{Introduction}

Paranasal sinus mucocoeles are slowly expanding cystic lesions leading to sinus expansion, bony erosion, and ultimately extension into the orbit, cranial cavity, and nasopharynx. ${ }^{1}$ Mucocoeles account for $4-8.5 \%$ of expanding orbital lesions ${ }^{2}$ with frontal and ethmoidal sinuses as the most common sites. Traditional management involves extirpation of the mucocoele lining and obliteration of the sinus. For those cases with significant orbital extension, a direct orbital approach has also been advocated. ${ }^{1}$

Over the past decade, intranasal drainage and marsupialization of mucocoeles using ESS has emerged as definitive management, with minimal morbidity and a low recurrence rate. ${ }^{3-12}$ With the advent of more advanced instrumentation, refined surgical techniques, and intraoperative imaging, even the more technically challenging frontal mucocoeles can be approached endoscopically.

The aim of this study was to evaluate the efficacy of ESS for sinus mucocoele with orbital involvement and assess the frequency with which a direct orbital approach is needed in such cases.

\section{Materials and methods}

A retrospective, consecutive case series of all paranasal sinus mucocoeles, with radiological evidence of orbital involvement, undergoing endoscopic sinus surgery (ESS) at the Departments of Surgery-Otolaryngology and Ophthalmology, Adelaide and Flinders Universities, over a 4-year period between 1998
${ }^{1}$ Oculoplastic \& Orbital Unit Department of Ophthalmology Royal Adelaide Hospital University of Adelaide Australia

${ }^{2}$ Department of SurgeryOtolaryngology Head \& Neck Surgery Adelaide and Flinders Universities Adelaide Australia

Correspondence: D Selva Oculoplastic and Orbital Clinic

Department of Ophthalmology Royal Adelaide Hospital

North Terrace

Adelaide 5000

South Australia

Tel: + 61882225222

Fax: + 61882225221

E-mail: Awestwoo@

mail.rah.sa.gov.au

Published online: 27 February 2004 
and 2002 is presented. All sinus mucocoeles with orbital extension referred to both departments were managed by ESS with no exclusions. A single surgeon (PJW) performed all surgery. Surgical procedures included simple drainage for frontoethmoidal mucocoele, modified Lothrop procedure with frontal drillout for frontal mucocoele, ${ }^{11,12}$ and the Landmarx protocol for lateral frontal mucocoeles and complex sinus anatomy to assist in visualization.

Patient profile data consisted of demographic details, past history of sinus surgery, concurrent nasolaryngopharyngeal comorbidities, the mucocoele site, and findings on preoperative computerized tomography.

All patients with orbital involvement underwent an ophthalmic evaluation and pre- and post- operative features (including ocular and nasal symptoms) were charted. The initial management and follow-up were also recorded.

In order to compare the number of symptoms and signs at presentation and during follow-up, patients were allocated a cumulative 'clinical score'. A maximum cumulative clinical score of 14 was possible (Table 1).

The outcome measures included change in symptoms and signs, patency of ostium, mucocoele recurrence or persistence, complications, additional surgery required, and duration of hospital stay.

Data were recorded on an Excel ${ }^{\mathbb{B}}$ spreadsheet.

Continuous data were analysed using a $t$-test and described as mean and standard deviation (mean \pm SD).

\section{Results}

A total of 15 patients (eight males, seven females) with 24 paranasal sinus mucocoeles underwent ESS. The mean age was $48 \pm 19$ (range 15-83) years and median followup was 15.5 (mean 16, range 1-40) months. All patients had radiological evidence of significant orbital involvement on $\mathrm{CT}$, with three cases demonstrating metaplastic bone formation at the margins of the mucocoele within the orbit (Figure 1). The mucocoeles were all well circumscribed within the orbit and none had an infiltrative appearance to the margin.

Table 1 Criteria for cumulative 'clinical score'

\begin{tabular}{lclc}
\hline Symptoms & Score & Signs & Score \\
\hline Anosmia & 1 & Visual loss & 1 \\
Epiphora & 1 & Globe displacement & 1 \\
Frontal pain & 1 & Lid swelling & 1 \\
Headache & 1 & Chorioretinal folds & 1 \\
Rhinorrhoea & 1 & Extraocular movement & 1 \\
& & restriction & \\
Nasal obstruction & 1 & Palpable mass & 1 \\
Posterior nasal drip & 1 & Ptosis & 1 \\
\hline
\end{tabular}
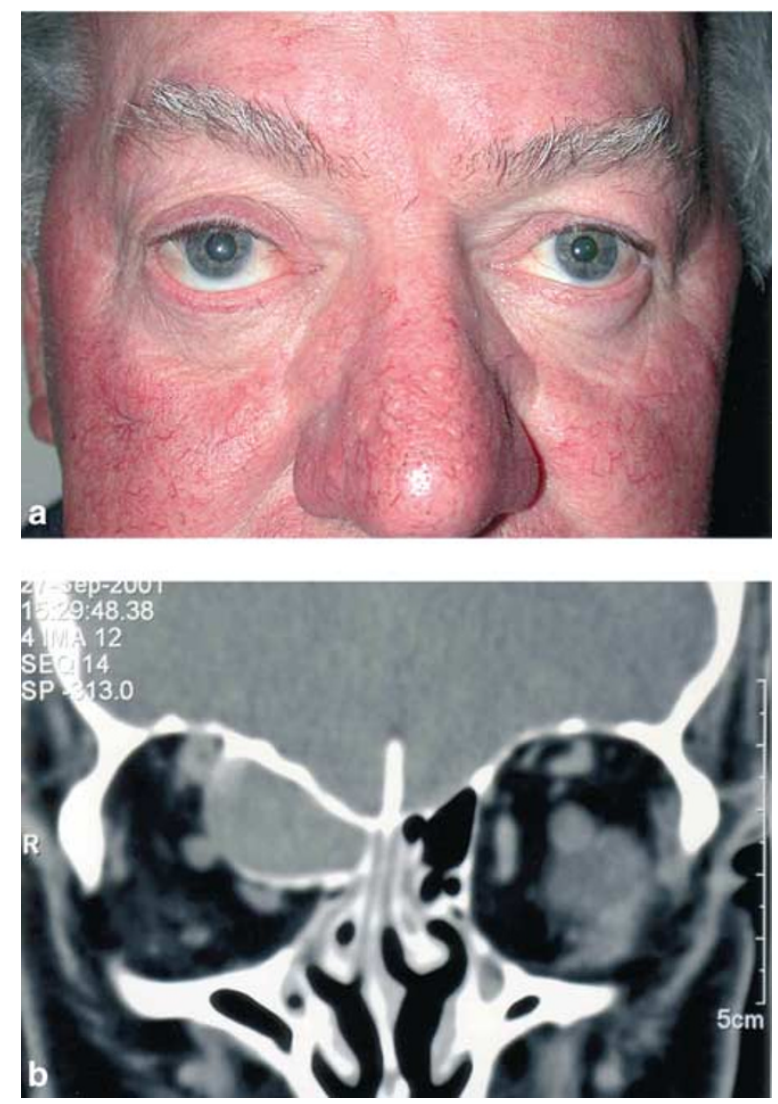

Figure 1 (a) Clinical photograph of a patient who presented with right-sided lateral globe displacement and proptosis. (b) Coronal computerized tomographic scan showed a well-circumscribed right frontoethmoidal mucocoele with metaplastic bone formation at the intraorbital margin.

The sites included frontal (42\%), frontoethmoidal (33\%), ethmoidal (8\%), and maxillary (17\%) sinuses. In the five patients with multiple lesions, at least one mucocoele demonstrated orbital involvement (Table 2).

Frontal pain and nasal obstruction were the main presenting symptoms, followed by diplopia and headache. Eleven patients $(73 \%)$ had globe displacement on presentation and four of these also had nonaxial proptosis. Visual acuity ranged between $6 / 6$ and no light perception in a patient with past history of traumatic optic neuropathy. One case with a frontal pyomucocoele presented with progressive reduction of vision, ptosis and periorbital swelling as a result of orbital cellulitis complicated by preseptal abscess. His visual acuity returned to $6 / 6$ within 2 weeks after the procedure. In terms of ophthalmic features, frontal and frontoethmoidal mucocoeles presented most commonly with inferolateral globe displacement and frontal pain, followed by diplopia and lid oedema. Ethmoidal mucocoele presented with lateral globe displacement, and of the three maxillary mucocoeles two were 
Table 2 Paranasal sinus mucocoele locations

\begin{tabular}{lr}
\hline Site of mucocoele & Number of mucocoele (total 24) \\
\hline Frontal & $10(41.7 \%)$ Left -5 , Right -5 \\
Frontoethmoidal & $8(33.3 \%)$ Left -5, Right -3 \\
Ethmoidal & $2(8.3 \%)$ Right -2 \\
Maxillary & $4(16.7 \%)$ Left -1 , Right -3 \\
Multiple mucocoeles & 5 patients \\
\hline
\end{tabular}

Table 3 Ophthalmic presentation of mucocoeles

\begin{tabular}{lcccc}
\hline Clinical features & Frontal & Ethmoidal & $\begin{array}{c}\text { Fronto- } \\
\text { ethmoidal }\end{array}$ & Maxillary \\
\hline Globe displacement & 6 & 2 & 5 & 1 \\
Diplopia & 3 & & 3 & \\
Lid oedema & 3 & 1 & 3 & \\
Nonaxial proptosis & 2 & & 2 & \\
Watery eye & 1 & & 1 & \\
Visual loss & 1 & & & \\
Ptosis & 2 & & & \\
Palpable orbital mass & 1 & & 1 & \\
\hline
\end{tabular}

Table 4 Nonophthalmic presentation of mucocoeles

\begin{tabular}{lcccc}
\hline Clinical features & Frontal & Ethmoidal & $\begin{array}{c}\text { Fronto- } \\
\text { ethmoidal }\end{array}$ & Maxillary \\
\hline Pain/headache & 6 & & 5 & \\
Nasal obstruction & 3 & 1 & 4 & 1 \\
Rhinorrhoea & 1 & & 3 & \\
Posterior nasal drip & 1 & 1 & 4 & \\
Anosmia & & 1 & 3 & \\
\hline
\end{tabular}

asymptomatic, being diagnosed on CT scan

(Tables 3 and 4).

Prior ENT history and co-morbidities are listed in

Table 5. Six patients had previously undergone endoscopic sinus surgery: frontal drillout (one), drainage of fronto-ethmoidal mucocoele (two), lateral rhinotomy (one), transitional cell papilloma excision (one) and antral washout (one). Seven patients had previously undergone external sinus surgery: frontoethmoidectomy (two), osteoplastic flap (two), Caldwell-Luc procedure (one), external rhinoplasty (one), frontal sinus trephine evacuation (one) and percutaneous mini-trephine for decompression of mucocoele (one). Three patients had undergone more than one nasal or sinus procedure previously.

\section{Treatment outcome}

All but one patient had resolution of ophthalmic symptoms and signs at the end of the follow-up period.
Table 5 Prior ENT history and co-morbidities

\begin{tabular}{lc}
\hline Prior ENT history and co-morbidities & Number of patients \\
\hline Chronic sinusitis & 12 \\
Fungal sinusitis & 3 \\
Nasal polyposis & 4 \\
Previous endoscopic sinus surgery & 6 \\
Previous sinus surgery external approach & 7 \\
Facial fractures & 2 \\
Prior neurosurgery-temporal & 2 \\
craniectomy for trigeminal rhizotomy (1), & \\
bilateral cranialization (1) & \\
Paranasal obstructive lesion-osteoma (1), & \\
inverting papilloma (1) & 2 \\
Asthma & 5 \\
Pyomucocoele & 1 \\
\hline
\end{tabular}

Cumulative clinical scores improved in all patients, with mean scores improving from $4.2 \pm 1.5$ (range $1-7$ ) to $0.4 \pm 0.7$ (range $0-2$ ) after surgery. In no cases did diplopia or decreased vision fail to resolve postoperatively. One patient with a large left frontal mucocoele had residual nonaxial proptosis due to persistent mucocoele. He had a past history of extensive traumatic fractures of both the anterior and posterior tables of his frontal sinuses requiring cranialization of both frontal sinuses. However, a significant part of the lateral left frontal sinus was not cranialized, but was separated from the nasal cavity. He subsequently presented with bilateral frontal mucocoeles.

Management of the left lateral frontal mucocoele was difficult due to the grossly disorganized frontal bone and the absence of a frontal recess on the left side. Under image guidance, a Rains drain was placed endoscopically to connect the lateral frontal sinus to the nasal cavity. He had residual postoperative proptosis which regressed after removal of blood clots from the Rains drain at 1 month follow-up. Post sinus toileting, he experienced intermittent proptosis that resolved spontaneously once every few months. In addition, four patients had incomplete resolution of nasal symptoms and signs, which were attributable to their underlying comorbidities such as fungal sinusitis or nasal polyposis. None of these cases had worsening of preoperative features.

Mucocoele recurred in two cases and persisted in one. Four patients required additional revision sinus surgery for narrowed frontal sinus ostium (two), mucocoele recurrence (two), and sinus toileting for adhesions and sinus debris (one). Among the four patients requiring additional surgery, two had multiple mucocoeles and fungal sinusitis, one had diffuse nasal polyposis, and one had complex sinus anatomy post neurosurgery. None required an external or direct orbital approach. Sinus 
ostia were patent in all cases excluding the patient with the Rains drain.

No major complications occurred, but minor, selflimiting complications were recorded in three cases: two epistaxis, one adhesion between middle turbinate and the lateral nasal wall, and one adhesion between the middle turbinate and septum.

The median duration of in-patient stay was 2 (mean 2.5 \pm 1.6 , range 1-6) days. Five patients were managed as day cases and three patients stayed overnight after the procedure. They were reviewed 2-3 days later as an outpatient. Those that remained as in-patients for longer did so for nonmedical reasons (patients lived too far for immediate outpatient follow-up).

\section{Discussion}

Mucocoeles with orbital involvement generally present with a noninfiltrating mass effect resulting in globe displacement, diplopia, proptosis, lid swelling, palpable mass, ptosis and reduced vision. Frontoethmoidal mucocoeles often present with swelling in the superonasal and medial canthal region, ptosis, and inferolateral globe displacement. Uncommonly, a sphenoidal mucocoele may lead to a compressive optic neuropathy. Maxillary mucocoeles may result in either upward globe displacement or enophthalmos secondary to bony erosion. Occasionally, as seen in one of our cases, when a mucocoele with orbital extension is complicated by infection, periorbital cellulitis may occur. ${ }^{1,2}$ Most patients $(93 \%)$ in our series had prior sinus surgery $(80 \%)$, chronic sinusitis $(80 \%)$ or trauma $(13 \%)$. Scarring of the sinus openings is the most common cause for mucocoele formation. The frontal and ethmoidal sinuses were the most commonly involved, which reflects the predisposition for their small sinus openings to be damaged during sinus surgery or trauma or by repeated infections with subsequent obstruction and mucocoele formation.

Our study represents the first to evaluate ophthalmic features and outcomes of paranasal sinus mucocoeles managed by ESS. The results indicate that the majority of sinus mucocoeles with orbital involvement can be managed effectively by endoscopic sinus surgery with minimal morbidity. All but one patient had full resolution of ophthalmic symptoms and signs postoperatively. The case with residual proptosis due to persistent mucocoele was a result of the technical difficulty in dealing with the grossly disorganized anatomy post-trauma. Most patients had patent sinus ostia and none had dural injury as has been previously reported..$^{10,13}$ None required external sinus or orbital surgery as definitive management even though most of our patients had significant orbital extension with $73 \%$ demonstrating globe displacement. These results suggest that a direct orbital approach is uncommonly, if ever, required. It has also been suggested that a direct orbital approach may be necessary to sharp dissect the mucocoele lining from the orbital soft tissue in those cases with adhesions due to recurrent inflammation. Our series included one case with recurrent orbital inflammation and one with orbital cellulitis, both of which had full resolution postoperatively. There were two mucocoele recurrences $(8 \%)$ which occurred in patients with diffuse nasal polyposis and chronic sinusitis complicated by fungal infection. The length of hospital stay was short, and compares favourably with external sinus surgery which has an average stay of 7 days. $^{3}$

Traditionally, treatment for paranasal sinus mucocoele involved complete removal of the sinus mucosal lining and obliteration of the sinus. In the frontal sinuses, some rhinologists have preferred osteoplastic flap with fat obliteration, as it is more cosmetically acceptable. However, this is an extensive procedure involving an external incision, periosteal elevation, and fracturing of the sinus floor in creation of the flaps. It also has a significant morbidity as stripping of the mucocoele lining occasionally leads to dural injury and CSF leaks. ${ }^{2,9,10}$ Obliteration of the involved sinus is not recommended if there is erosion of the sinus bony wall with extension of the mucocoele either intracranially or into the orbit. The mucosa lining the mucocoele becomes adherent to either the dura or the orbital periosteum and cannot be removed during the surgery without significant risk of injury to the adjacent structures. If mucosa is left behind and the sinus obliterated, a recurrence of the mucocoele is highly likely. ${ }^{9}$ As most cases of symptomatic mucocoele have erosion of the bony sinus wall, obliteration of the sinus should not be considered. The alternative is to ensure that the mucocoele drains into the nasal cavity. As was seen in this series of patients, laterally based frontal sinus mucocoeles can provide a significant surgical challenge to re-establish and maintain a patent opening between the mucocoele and the nasal cavity, with a number of patients requiring revision surgery to ensure continued patency. The follow-up of an obliterated sinus can also be unreliable as imaging may not be able to distinguish an obliterated sinus from a recurrent mucocoele. ${ }^{3,9}$ In addition, it has been shown that mucosa of a mucocoele does not lose normal respiratory epithelium. ${ }^{14}$ Histological examination of frontal mucocoele lining 6 months after marsupialization revealed areas of normal ciliated pseudostratified epithelium, ${ }^{15}$ and follow-up endoscopic examination has also found well-mucosalized cavities following 
marsupialization. ${ }^{6}$ These findings suggest that there is no added benefit in attempting to remove the mucocoele lining.

Our study adds to the number of publications advocating ESS as a safe and effective approach for mucocoele management. Har et $a l^{10}$ reported the largest series of 103 patients (108 mucocoeles) with a median follow-up of 4.7 years and a recurrence rate of $0.9 \%$. This also represents the longest follow-up for endoscopically managed mucocoeles. Recurrence of mucocoele after osteoplastic and obliteration procedures was 19\% (nine out of 47 patients) in a retrospective study, ${ }^{16}$ whereas in two endoscopic series, ${ }^{3,7}$ the recurrence rate was zero percent with minimal complications. It is important to note that, in these latter two studies, patients thought not suitable for an entirely endosocopic approach due to complex sinus anatomy were managed with an external approach or a combined endoscopic and external approach. However, despite the addition of an external incision, the principle of mucocoele marsupilization into the nasal cavity was maintained and obliteration was rarely performed.

In our study, the cumulative clinical score was devised to quantify symptoms and examination findings to enable objective comparison pre-and post-ESS to assess efficacy of the procedure. An improvement in the scores was attributed to resolution of the mucocoele. While we recognize the possible shortcomings of such scoring (nonspecific nature of some symptoms and signs), they do provide an indication of mucocoele resolution and are an alternative to repeat radiology which would generally be unnecessary in an asymptomatic patient.

Endoscopic sinus surgery should be considered as a treatment option for paranasal sinus mucocoele with orbital involvement and a direct orbital approach should not be required. Marsupialization of the mucocoele into the nose is effective in improving ophthalmic symptoms and signs due to mucocoeles, although longer term follow-up is required to fully evaluate outcomes.

\section{References}

1 Rootman J, Allen L. Structural lesions. In: Rootman J (ed). Diseases of the Orbit, 2nd edn. Lippincott Wiliams \& Wilkins: Philadelphia, 2003, pp 425-431.

2 Haik BG, Amedee RG. Ophthalmic manifestations of mucoceles. In: Bosniak S, ed. Principles and Practice of Ophthalmic Plastic and Reconstructive Surgery. WB Saunders: Philadelphia, 1996, pp 1014-1022.

3 Kennedy DW, Josephson JS, Zinreich SJ, Mattox DE, Goldsmith MM. Endoscopic sinus surgery for mucoceles: a viable alternative. Laryngoscope 1989; 99: 885-895.

4 Schaefer SD, Close LG. Endoscopic management of frontal sinus disease. Laryngoscope 1990; 100: 155-160.

5 Benninger MS, Marks S. The endoscopic management of sphenoid and ethmoid mucoceles with orbital and intranasal extension. Rhinology 1995; 33: 157-161.

6 Har-EL G, Balwally AN, Lucente FE. Sinus mucoceles: is marsupialization enough? Otolaryngol Head Neck Surg 1997; 117: 633-640.

7 Lund VJ. Endoscopic management of paranasal sinus mucocoeles. J Laryngol Otol 1998; 112: 36-40.

8 Ikeda K, Takahashi C, Oshima T, Suzuki H, Satake M, Hidaka H, Takasaka T. Endonasal endoscopic marsupialization of paranasal sinus mucocoeles. Am J Rhinol 2000; 14: 107-111.

9 Har-EL G. Transnasal endoscopic management of frontal mucoceles. Otolaryngol Clin North Am 2001; 34: 243-251.

10 Har-EL G. Endoscopic management of 108 sinus mucoceles. Laryngoscope 2001; 111: 2131-2134.

11 Wormald PJ. Salvage frontal sinus surgery: the endoscopic modified Lothrop procedure. Laryngoscope 2003; 113: 276-283.

12 Wormald PJ, Ananda A, Nair S. The modified endoscopic Lothrop procedure in the treatment of complicated chronic frontal sinusitis. Clin Otolaryngol 2003; 28: 215-220.

13 Stennert E. Rhino-frontal sinuseptotomy (RFS): a combined intra-extra nasal approach for the surgical treatment of severely diseased frontal sinuses. Laryngoscope 2001; 111: 1237-1245.

14 Lund VJ. Fronto-ethmoidal mucocoeles: a histopathological analysis. J Laryngol Otol 1991; 105: 921-923.

15 Har EL, Dimaio T. Histologic and physiologic studies of marsupialized sinus mucocoeles. J Otolaryngol 2000; 29: 195-198.

16 Bordley JE, Bosley WR. Mucocoeles of the frontal sinus: causes and treatment. Ann Otol Rhinol Laryngol 1973; 82: 696-702. 